\section{Resolution of Digital Photomicrographs from Scanned Film}

Theodore M. Clarke, Metallurgical Failure Analysis Consultant tclarke@kuntrynet.com

Some may have read my previous articles in Microscopy Today and know that I am a strong proponent of digital imaging for photomicrographs and photomacrographs. I require that the digital images match the resolution and field size of traditional film images, with the $4 \times 5$ Polaroid film most commonly used in metallurgical laboratories where I worked before early retirement ${ }^{1}$. My development of an affordable universal student microscope for home microscopy resulted in a need for color digital images that would meet my requirements at an affordable cost $^{2}$. This need was met by recording on $35 \mathrm{~mm}$ film with subsequent scanning of selected film images to a Kodak Master Photo CD. More recently I purchased a CanoScan FS2710 film scanner for digitizing my family's collection of slides and negatives for saving on CD's. This article will review how I arrived at my requirements and how well they were met by digital cameras and by film scanning.

\section{MICROSCOPE RESOLUTION}

The definition of the spatial resolution of the microscope begins with the diffraction pattern image of two nearly adjacent point sources of light formed through a circular aperture. The point sources are separated in object space so that the first minima of one pattern coincides with the central maxima of the other diffraction pattern. This is the Rayleigh criterion of resolution. The image of these two point sources is the sum of the two pattern intensities as shown in Figure $1^{3}$. Recording a straight line series of overlapping Airy discs at the Rayleigh limit with a CCD would require at least two pixels per Airy disk with the pixels centered on the maxima and minima corresponding to a pixel size of one fourth the Airy disc diameter. The delta $Y$ separation in the image can be related back to the separation of the point sources in object space through the application of Abbe's sine condition for a lens free of spherical aberration. This objective lens resolution is given in Figure 2.

\section{FIELD SIZE}

The recording of photomicrographs requires consideration of the field size limitations of the microscope. The compound microscope optics produce an intermediate image which was

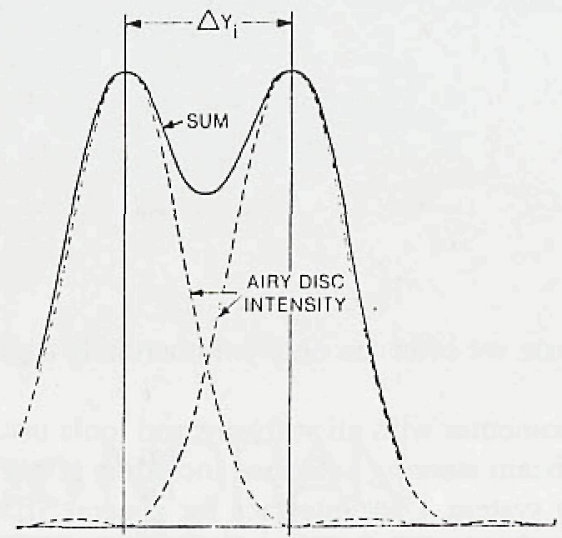

INTENSITY SUM OF TWO AIRY DISCS SEPARATED SO THAT THE MAXIMUM OF ONE FALLS ON THE FIRST DARK MINIMUM OF THE OTHER

Figure 1: Diffraction pattern image of two closely spaced point sources of light just resolved. conventionally enlarged for viewing with a $10 \mathrm{X}$ eyepiece and projected with $10 \mathrm{X}$ enlargement for recording on $4 \times 5$ film or with $2.5 \mathrm{X}$ enlargement for recording on $35 \mathrm{~mm}$ film. Until recently the intermediate field size was limited to about $18 \mathrm{~mm}$. Modern research microscopes may now have an intermediate field size diameter as large as $26 \mathrm{~mm}$ in diameter as shown in Figure 3 . The size of the rectangle in the intermediate image enlarged to form the photomicrograph is important in determining the physical size of the CCD array and relay lens magnification for digital recording. The intermediate field size traditionally recorded on $4 \times 5$ film is a $8.9 \times 11.4$ $\mathrm{mm}$ rectangle as shown in Figure 3 . Recording a $17.8 \times 17.8 \mathrm{~mm}$ square format image from the $26 \mathrm{~mm}$ intermediate image diameter would make good use of the modern optics when necessary.

\section{FINAL PRINT RESOLUTION}

Resolution of the final photomicrograph is rarely mentioned in quantitative terms. The viewing and recording optics of the light microscope have been consistent with Abbe's definition of useful magnification, which is related to the resolving ability of normal vision when the photomicrograph is viewed from $250 \mathrm{~mm}$. Images from the lower power objectives generally meet an Abbe magnification criterion of 500X the NA of the objective. An Abbe criterion of $1000 X$ the NA is commonly used with the highest power objectives so that the finest detail resolvable by the objective can be

\section{Microscope Objective Resolution}

$$
\begin{aligned}
& \text { Object Resolution }=\frac{.61 \lambda}{\mathrm{N} . \mathrm{A} .} \\
& \text { Setting } \lambda=5.5 \times 10^{-4} \mathrm{~mm} \\
& \frac{1}{\text { Object Resolution }}=\text { lines } / \mathrm{mm} \text { Resolution } \\
& \text { Object Resolution }=3000 \text { N.A. lines } / \mathrm{mm} \\
& \text { Example: } 4 \text { X } .10 \text { N.A. Objective } \\
& \text { Object Resolution }=300 \text { lines } / \mathrm{mm}
\end{aligned}
$$

Figure 2: Equation for resolution with sample calculation.

\section{Intermediate Image Size Comparison}

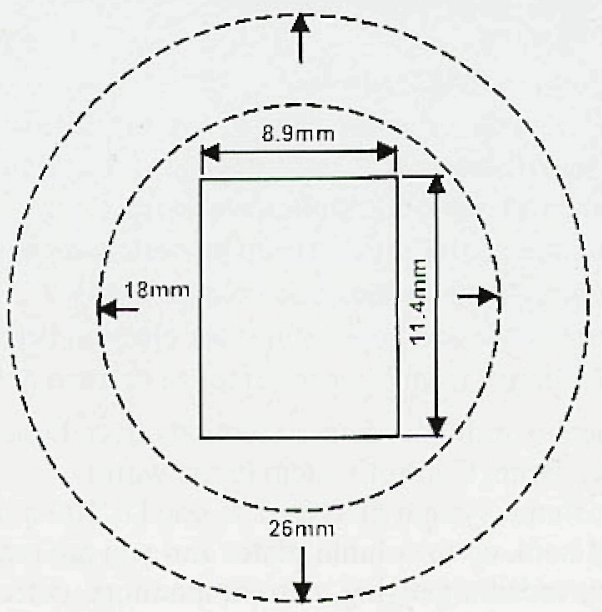

Figure 3: Intermediate image field size comparison of modern with older microscope technology and the rectangular field portion traditionally recorded on $4 \times 5$ film.

\section{Continued on following page}


Resolution of Digital Photomicrographs from

Scanned Film

Continued from preceding page

easily seen when higher NA is not available. Images from the lower power objectives place more demand on the resolution of the film or CCD as indicated in the analysis given in Figure 4. Assuming a traditional $10 \mathrm{X}$ enlargement of the intermediate image to achieve photomicrograph resolution of between 6 and 3 line pairs per $\mathrm{mm}$, the intermediate image resolution ranges between a corresponding 60 and 30 line pairs per $\mathrm{mm}$.

\section{PIXEL ARRAY SIZE REQUIREMENT}

A minimum pixel array size that is needed to achieve 6 line pair per $\mathrm{mm}$ resolution in the image area of a $4 \times 5$ Polaroid film print is derived in Figure 5 . The calculation agrees well with the now widespread use of $1280 \times 1024$ through $1600 \times$ 1200 pixel CCD cameras for scientific imaging. The calculation assumes that the three-color values for each pixel are not interpolated as with consumer product cameras having a color mosaic filter pattern over the pixels, which reduces spatial resolution by at least $30 \%$. Non-interpolated pixel values can be obtained by many approaches. The most common noninterpolated color capture is by sequential capture of red, green and blue images using a color filter wheel or tunable liquid crystal filter. Film scanners and some color digital cameras record the image by scanning the optical image with a red, green and blue filtered trilinear array.

\section{RESOLUTION TEST PATTERNS}

Measuring the resolution capability of a digital camera or film scanner for photomicrographs ideally requires a micro- scope resolution test slide. The lowest magnification objective is commonly a $5 \times 0.10 \mathrm{NA}$ ( $4 \times 0.10 \mathrm{NA}$ used with a $1.25 \mathrm{X}$ tube factor in some Zeiss microscopes). This lens should resolve 300 line pairs per $\mathrm{mm}$ in object space forming an intermediate image with 60 line pairs per mm resolution. A 3" $\times 3$ " chrome on glass resolution test chart with patterns from $1 / 4$ line pair per $\mathrm{mm}$ to 600 line pairs per mm is available and quite costly. I previously used this test chart with a 0.10 NA Zeiss objective and verified the theoretical resolution with both the MegaPlus 1.6i/AB digital camera and recording on Polaroid Type 55 film. Low cost test patterns with a

$$
\begin{aligned}
& \text { Photomicrograph Resolution } \\
& \text { Object Resolution }=\frac{1}{3000 \text { N.A }} \\
& \text { Abbe Criterion of Useful Magnification } \\
& 500 \text { N.A. } \leq \text { Mtctal } \leq 1000 \text { N.A. } \\
& \text { Using } 500 \text { N.A. }=\text { Mrata } \\
& \text { Final Image Resolution }=\text { Mtotal } \times \text { Object Resolution } \\
& \text { (in } \mathrm{mm} \text { ) } \\
& \begin{array}{l}
\text { Final Image Resolution }=500 \text { N.A. } \times \frac{1}{3000 \text { N.A. }} \text { (in mm) }
\end{array} \\
& \text { Final Image Resolution }=1 / 6 \mathrm{~mm} \text { For } 500 \text { N.A. Criterion } \\
& 3 \leqslant \text { Final Image Resolution } \leqslant 6 \text { in Lines } / \mathrm{mm}
\end{aligned}
$$

Figure 4: Derivation of photographic print resolution from Abbe's criterion. 
small field size with line patterns covering a range of 300 to 3000 line pairs per $\mathrm{mm}$ could be made by electron lithography but are not available because of lack of market demand. An alternative test method is to use a high resolution macro lens on the camera to image a pattern like the NBS Microcopy Resolution Test Chart in the $89 \times 114 \mathrm{~mm}$ field size of a $4 \times 5$ Polaroid film print for a $1280 \times 1024$ pixel CCD sensor $(89 \times 133 \mathrm{~mm}$ field for a $1534 \times$ $1024 \mathrm{CCD}$ sensor or film scan of $35 \mathrm{~mm}$ format) as done for Figures $6 \& 7$.

\section{DIGITAL CAMERA AND KODAK PHOTO CD RESOLUTION}

The test result for the Mavica camera demonstrates the significant loss in spatial resolution due to the color mosaic filter over the CCD sensor when compared with the grayscale capture by the MegaPlus camera. The pattern results with the MegaPlus camera demonstrate that line patterns oriented orthogonal to the pixel array are more difficult to record without an alias line problem. The alias lines are a moiré fringe effect from close alignment of the pixel spacing with the line patterns in the optical image when the spatial frequency of the line patterns approach the pixel spacing. The moire effect was accentuated with the MegaPlus results because the original images were high pass filtered to improve the apparent resolution. The position of the test pattern during imaging was purposely adjusted to avoid this effect for the orthogonal orientation of the 5.6 line pairs per $\mathrm{mm}$ pattern. The orthogonal patterns from the Photo CD scans show less evidence of this effect, perhaps mainly because they were not sharpened. The solution to the alias line problem is to capture the same image with a larger number of pixels. Since my home microscope and the older Zeiss Universal microscope I used before retirement have a maximum intermediate image field diameter of $18 \mathrm{~mm}$, a $1534 \times 1024$ pixel array meets my requirements for resolution and field size. The $3000 \times 2000$ pixel resolution result indicates that the $35 \mathrm{~mm}$ film to digital method using this Kodak Photo $C D$ file size cropped to $2048 \times 2048$ pixels should be adequate to cover an $18 \times 18$ $\mathrm{mm}$ portion of a $26 \mathrm{~mm}$ intermediate image field diameter for those fortunate enough to own a modern research microscope. The 11 line pair per $\mathrm{mm}$ pattern just resolved in the $3000 \times 2000$ pixel file corresponds to 40 line pairs per $\mathrm{mm}$ in the film image. The blurring effect of limited film resolution is showing up at this higher spatial frequency.

\section{CANON FILM SCANNER RESOLUTION}

Film scanners for use with fast PC's with 8 megabyte graphics cards, $1600 \times 1200$ pixel monitors and $C D$ writers are now affordable for home use. I have a large collection of family $35-\mathrm{mm}$ slides and negatives and wanted to digitize them with storage on CD's for distribution to the younger members of the family so their past will not be lost. The cost savings of owning my own film scanner versus having the film images scanned to Kodak Master Photo

\section{Estimated Pixel Array Requirement}

Polaroid® Image Size: $89 \mathrm{~mm}$ by $114 \mathrm{~mm}$

Image Resolution Criterion: 6 lines or cycles $/ \mathrm{mm}$

2 pixe/s/cycle $\times 6 \mathrm{cycles} / \mathrm{mm} \times 89 \mathrm{~mm}=1070$ pixe/s

2 pixels $/$ cycle $\times 6 \mathrm{cycles} / \mathrm{mm} \times 114 \mathrm{~mm}=1370$ pixe $/ \mathrm{s}$

Figure 5: Estimate of minimum pixel array size to match the resolution of a Polaroid $4 \times 5$ film print recorded with an Abbe magnification of 500 times the NA of the objective.

\section{want one?}
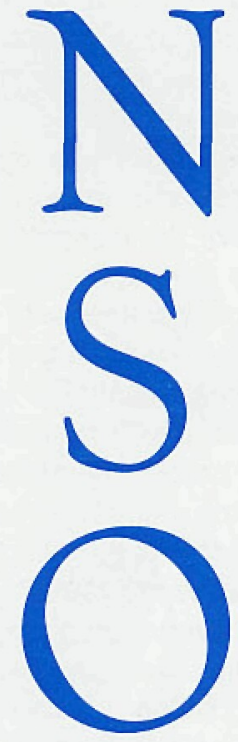

M

\section{thought sol}
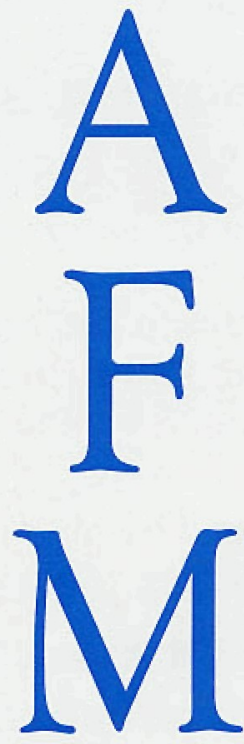

www.thermomicro.com www.spmprobes.com

\section{visit us!}


Resolution of Digital Photomicrographs from

Scanned Film

Continued from preceding page

CD's justified purchase of a Canon CanoScan FS2710 film scanner. A maximum resolution scan for this scanner is $3888 \mathrm{X}$ 2720 pixels. This pixel array size is not adequate to record the finest spatial frequencies in very high quality slides and negatives, but should achieve about 52 line pairs per $\mathrm{mm}$ resolution. Some $35 \mathrm{~mm}$ film images exceed 63 line pairs per $\mathrm{mm}$ resolution. The need to digitize the highest spatial frequencies on 35 $\mathrm{mm}$ film is met by the Kodak Professional Photo CD $6000 \mathrm{X}$ 4000 pixel scans. Film scanners with this resolution are now available, but quite expensive for home use. Owning the CanoScan film scanner is a big advantage for my low budget, home microscopy. I can record on $35 \mathrm{~mm}$ color negative film and use locally available 1 hour processing of the film prior to scanning it at home. Scanning the negatives rather than photographic prints from the negatives avoids the problem of variable print quality, which the mass market probably would not notice. The disadvantage is that I have to bracket my exposures to be sure of a good film image to scan. Naturally I have compared the resolution of the Canon scanner, using the same resolution test slide, with the Kodak Photo $C D$ results in Figure 6 . The finest pattern on the NBS test chart just resolved on the film is 14 line pairs per $\mathrm{mm}$ pattern corresponding to 51 line pairs per $\mathrm{mm}$ on the slide. PhotoShop LE comes with the scanner software and was used to downsize the large file size to $1940 \times 1360$ and $1534 \times 1024$ pixel files. A photomontage of the finest patterns in these files is shown in Figure 7. It is important to note that the other than $2: 1$ downsizing used to obtain the $1534 \times 1024$ pixel

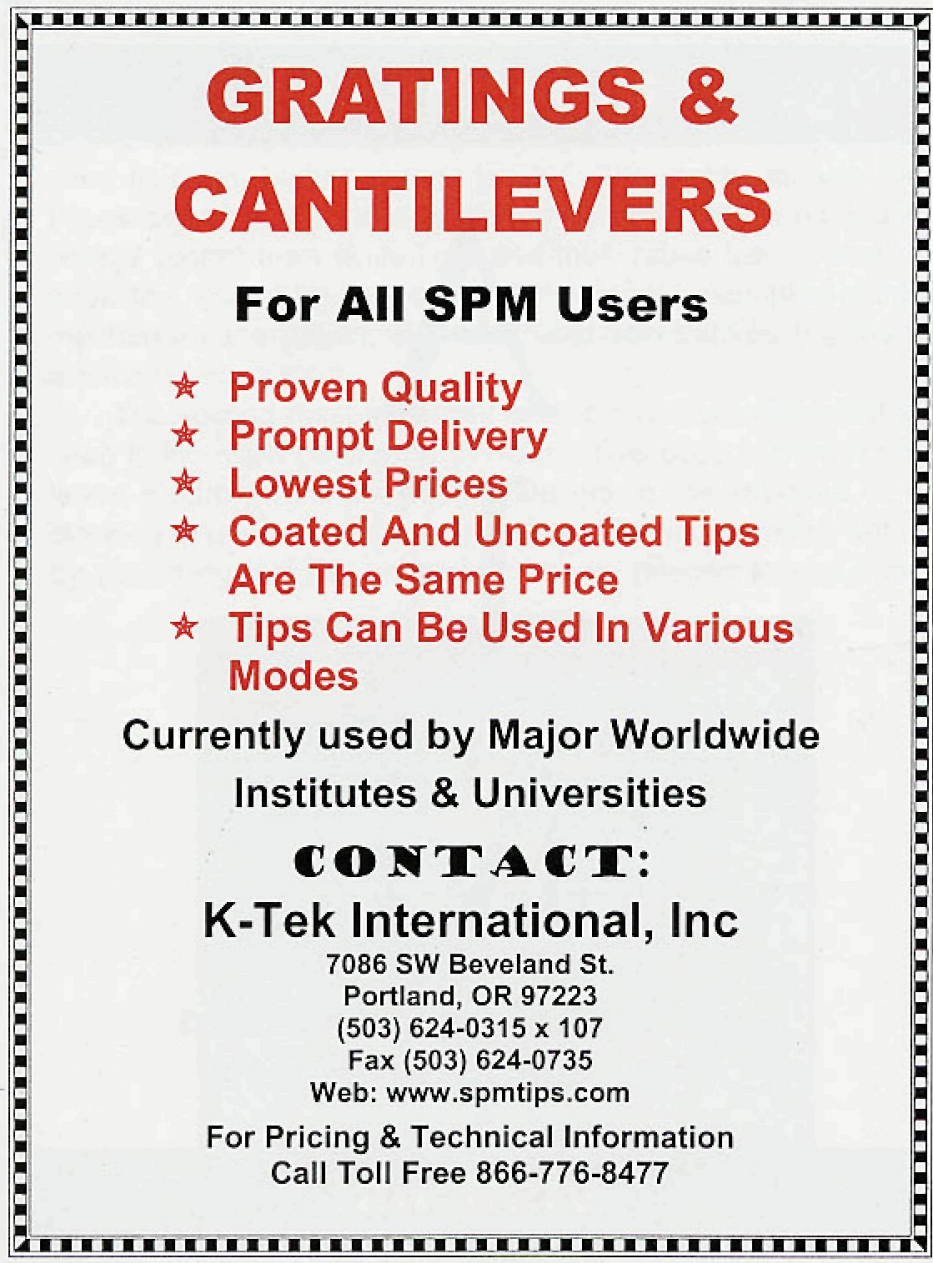

file introduces noticeable alias line artifacts less evident than in the Photo CD result shown in Figure 6.

Film-to-digital and direct digital capture, with a digital camera, can clearly be acceptable methods for obtaining high quality digital photomicrographs that match the field size and resolution of traditional photomicrograph prints. Unfortunately there are no national or international standards dealing with this important transition in technology. The Photographic and Imaging Manufacturers Association (PIMA) is participating in an ISO TC42 effort to develop such standards ${ }^{4}$. Their preliminary efforts concentrate on using resolution test pattern of their own design and alias effects are considered. Alias effects are inherent in the digital capture, but need to be worsened in subsequent image downsizing and desktop publishing. Understanding the operating principles of the light microscope and using them to obtain an optimum optical image for recording is vital for quality digital images. Unfortunately the users who think software can "fix" the resulting defects their images do not appreciate this requirement.

1. Clarke, T.M., "Digital Imaging in the Materials Engineering Laboratory." THE MICROSCOPE, 1998, 46(2), 85-100.

2. Clarke, T.M., "Building an Affordable Universal Student Microscope, "THE MICROSCOPE, 2000, 48(1), 19-39

3. Moller, K.D., OPTICS, University Science Books, Mill Valley, CA, 1988.

4. Edwards, Parulski \& Holm, "Setting Standards-Developing Standards

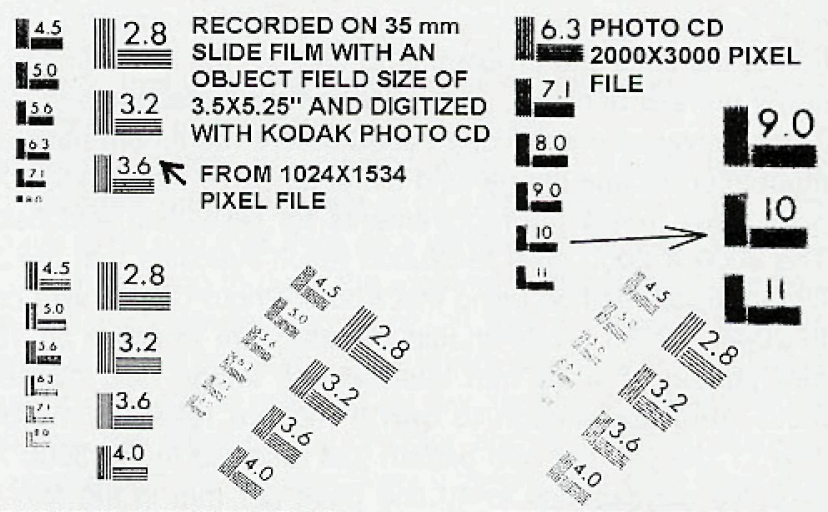

RECORDED WITH MEGAPLUS 1.6i/AB CAMERA IN A $3.5 \times 5.25^{\prime \prime}$ OBJECT FIELD SIZE WITH A $1024 \times 1534$ PIXEL ARRAY SENSOR
RECORDED WITH SONY FD88 MAVICA CAMERA IN AN OBJECT FIELD SIZE OF $3.25 \times 4.5 "$ WITH A $960 \times 1280$ PIXEL ARRAY SENSOR
Figure 6: Comparison of digital camera resolution with Kodak Photo $\mathrm{CD}$ resolution using the highest resolution portions cropped from the original images.
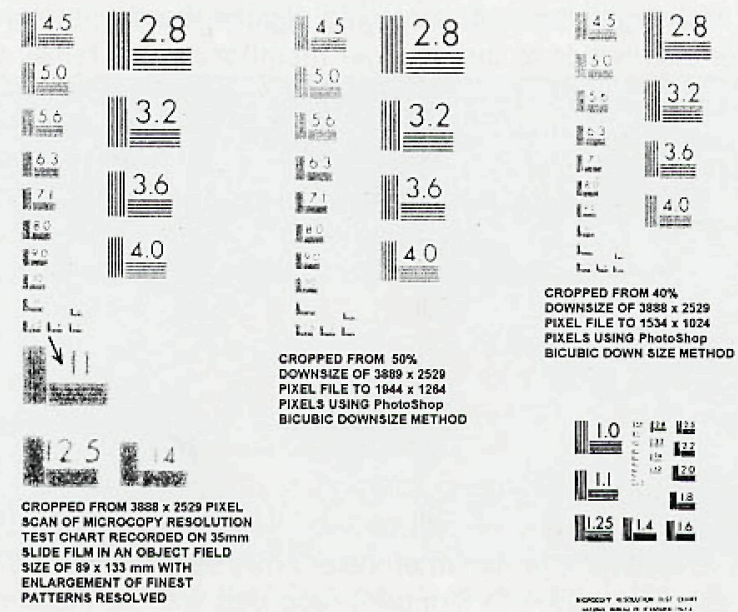

Figure 7: Resolution test results for the Canon CanoScan FS2710 film scanner. 